\title{
Transmit and Receive Circular Array Pattern Synthesis for Radar Applications
}

\author{
W. Mark Dorsey, Senior Member, IEEE, and Dan P. Scholnik
}

\begin{abstract}
Circular arrays are an appealing if underused option for radar systems that require $360^{\circ}$ coverage in azimuth. They require no mechanical scanning and do not suffer from the high-angle scan loss and beam broadening of linear and planar arrays. The circular geometry, along with suitable pattern synthesis techniques, enables some unique capabilities. On transmit they provide the flexibilty to synthesize a continuum of beam shapes, from omnidirectional to sector to high gain. On receive, simultaneous high-gain beams can be formed to cover any desired azimuth footprint. In this paper we explore the capabilities of circular arrays through a series of pattern synthesis design examples.
\end{abstract}

Index Terms-Antenna phased arrays, Optimization, Beamforming arrays, Radar

\section{INTRODUCTION}

$\mathbf{R}$ ADAR systems requiring full visibility of the azimuth plane require an antenna system that offers $360^{\circ}$ of scanning. A uniform circular array (UCA) consists of $N$ antenna elements arranged around a circle of radius $R$ with angular spacing $2 \pi / N$ between adjacent elements. UCAs offer full coverage of the azimuth plane with either a more-orless omnidirectional radiation pattern or with electronically steerable directional beams. The directional beams can be electronically scanned in the azimuth plane without significant changes to the beamwidth and sidelobe level (SLL)[1], [2].

The ability to support functions requiring omnidirectional antennas as well as those needing directional patterns make UCAs desirable for platforms providing full $360^{\circ}$ coverage of the azimuth plane. In a traditional design, these functions would require multiple apertures: typically a suite of omnidirectional antennas tuned to appropriate frequency bands combined with planar/linear arrays for the directional patterns. Oftentimes, multiple planar/linear arrays—or a rotating planar/linear array - are needed for each function to facilitate electronic scanning throughout the azimuth plane.

An application of a UCA to radar systems is discussed in [3]. In this type of arrangement, the circular array can form both the sum and difference beams required for a monopulse radar system while also forming an omnidirectional pattern that can be used as the control channel for discriminating targets outside of the main beam. Circular arrays have been proposed for passive coherent radar due to their ability to form beams in arbitrary directions [4]. The UCA geometry also lends itself conveniently to direction of arrival (DOA)

Manuscript received March 16, 2016. This work was supported by the NRL Base Program.

The authors are with the Radar Division, U.S. Naval Research Laboratory, Washington, DC 20375, e-mail: mark.dorsey@nrl.navy.mil algorithms [5] to support locating and tracking the transmitter of opportunity.

In this paper we focus on the array synthesis problem for both transmit and receive, as this is what most clearly distinguishes circular arrays from the more-common linear arrays and illustrates the utility for radar. On receive, digital beamforming allows multiple simultaneous beams to be synthesized, and thus arbitrary regions can be covered with high-gain beams. We use the approach of [6] to formulate the receive beamforming problem as a second-order cone program (SOCP) that trades off taper-loss and sidelobe levels. On transmit circular arrays can form both high-gain and sector beams, similar to linear arrays. Sector beams are often used to improve search performance by illuminating multiple receive beams simultaneously [7]. Unlike linear arrays, circular arrays can also be used to synthesize omnidirectional patterns in azimuth, allowing for ubiquitous-radar [8] operation. Heuristic approaches exist to generate sector and omnidirectional patterns, and we use these to initialize a phase-only patternsynthesis algorithm adapted from [9] to optimize the patterns, potentially including transmit nulling. These techniques will be illustrated through example designs on a 31 element array.

\section{Pattern Synthesis}

The array pattern for an arbitrary array of $N$ elements

$$
\vec{F}(\mathbf{k})=\sum_{n=1}^{N} w_{n} \vec{f}_{n}(\mathbf{k})
$$

can be defined in terms of complex excitations $\left\{w_{n}\right\}$ and complex embedded element patterns $\left\{\vec{f}_{n}(\mathbf{k})\right\}$, which are functions of vector wavenumber $\mathbf{k}$, as discussed in [10]. For a UCA, the embedded pattern of each element can be related to that of element $N$, serving here as the prototype, via

$$
\vec{f}_{n}(\mathbf{k})=\mathbf{R}^{n} \vec{f}_{N}\left(\mathbf{R}^{-n} \mathbf{k}\right)
$$

for some fixed $3 \times 3$ rotation matrix $\mathbf{R}$. To ensure uniform element distribution and that no elements are duplicated, $\mathbf{R}$ should have the property that $\mathbf{R}^{M}=\mathbf{I}$ if and only if $M$ is a multiple of $N$.

Including directional elements in a circular array improves the patterns that can be formed at the expense of increased complexity in the synthesis techniques that must be applied [11]. Pattern synthesis for a circular array of directional elements can be somewhat more involved than for linear or planar arrays since array-factor design techniques are no longer applicable and each embedded element pattern must be included. In this section we discuss circular array-pattern 
synthesis techniques to design complex-weight excitations $\left\{w_{n}\right\}$ that satisfy a specified set of far-field constraints for both receive and transmit.

\section{A. Receive Pattern Synthesis}

On receive, we assume that we have full control over the complex weights applied to each element, and seek to design directional beams that optimally trade off signal-to-noise ratio (SNR) and sidelobe level (SLL). This is a convex problem, and in [6] it was shown how to formulate such a design as a second-order cone program (SOCP). SOCP is an efficient, structured form of convex optimization that minimizes a linear objective subject to linear and convex quadratic constraints. Several SOCP solvers are freely available [12]-[14]. Here we develop a SOCP formulation in the spirit of [6] but with a different normalization.

For our design we wish to define terms proportional to mainbeam signal and noise in the array output, in order to define SNR, as well as define an expression for sidelobe energy. These are considered in turn.

1) Mainbeam signal: The output signal of the UCA beamformer due to an incoming plane wave from direction $\mathbf{k}_{\mathrm{mb}}$ with polarization unit-vector $\hat{c}$ is proportional to $\vec{F}\left(\mathbf{k}_{\mathrm{mb}}\right) \cdot \hat{c}$, which can also be written as the inner-product $\mathbf{w}^{T} \mathbf{f}_{\mathrm{mb}}=\left\langle\mathbf{f}_{\mathrm{mb}}, \mathbf{w}^{*}\right\rangle$, where $\mathbf{f}_{\mathrm{mb}}$ is the vector of co-pol element patterns $\left\{\vec{f}_{n}\left(\mathbf{k}_{\mathrm{mb}}\right) \cdot \hat{c}\right\}$ in the direction $\mathbf{k}_{\mathrm{mb}}$ and $\mathbf{w}^{*}$ is the conjugate of the column vector of complex excitations $\left\{w_{n}\right\}$.

2) Receiver Noise and SNR: We will assume uncorrelated white noise at each element with identical noise densities, so that output noise power is proportional to $\|\mathbf{w}\|^{2}$. The noise and signal expressions can be related through the Cauchy-Schwarz inequality as $\left|\mathbf{w}^{T} \mathbf{f}_{\mathrm{mb}}\right|^{2} \leq\|\mathbf{w}\|^{2}\left\|\mathbf{f}_{\mathrm{mb}}\right\|^{2}$, or rearranging to explicitly show SNR,

$$
\frac{\left|\mathbf{w}^{T} \mathbf{f}_{\mathrm{mb}}\right|^{2}}{\|\mathbf{w}\|^{2}} \leq\left\|\mathbf{f}_{\mathrm{mb}}\right\|^{2} .
$$

From this we can see that SNR is maximized when $\mathbf{w}^{*}$ and $\mathbf{f}_{\mathrm{mb}}$ are colinear. For a linear or planar array with identical elements this would simply be a complex exponential vector, but for a UCA the max-SNR weights will have significant amplitude variation. We will revisit this expression in the following section.

3) Sidelobes: In the mainbeam we were only interested in the output due to a known polarization, but for sidelobe signals we wish to constrain the pattern for all polarizations. For this purpose we can use the norm $\left\|\vec{F}\left(\mathbf{k}_{s}\right)\right\|$ of the vector pattern.

Finally, we can put the above together to get our SOCP:

$$
\begin{aligned}
\max _{\mathbf{w}, \Lambda} & \operatorname{Re}\left\{\mathbf{w}^{T} \mathbf{f}_{\mathrm{mb}}\right\} \\
\text { s.t. } & \|\mathbf{w}\| \leq 1 \\
& \left\|\vec{F}\left(\mathbf{k}_{s}\right)\right\| \leq \delta, \quad s=1,2, \ldots, s_{\max } .
\end{aligned}
$$

Here the objective (3) to be maximized is the real part of the co-pol component of the array pattern in the mainbeam direction. This effectively maximizes $\left|\mathbf{w}^{T} \mathbf{f}_{\mathrm{mb}}\right|$ as all the expressions in the SOCP are invariant to complex rotation of $\mathbf{w}$ and the corresponding imaginary part will be zero at optimality due to the constraints. Constraint (4) limits the white noise gain of the array to unity, normalizing the array pattern to indicate SNR gain relative to an isotropic radiator. Constraint (5) limits the peak value of the combined co-pol and cross-pol sidelobes over the set of directions $\left\{\mathbf{k}_{s}\right\}$ to a constant $\delta$. This constraint, when active, results in deviations from the ideal SNR derived above. Thus the choice of sidelobe locations $\left\{\mathbf{k}_{s}\right\}$ and level $\delta$ results in an inherent tradeoff with SNR.

\section{B. Transmit Pattern Synthesis}

Transmit pattern synthesis differs from receive synthesis in a couple of key ways. Simultaneous independent transmit beams are generally impractical, and instead multiple receive beams are usually illuminated with a single broad transmit beam. Broad-beam designs, in turn, are often nonconvex and suffer from many poor local minima [15]. Further, transmit hardware often places restrictions on the allowable complex weights, either upper-bounding their magnitude (representing the power limit of each transmitter) or fixing their magnitude for amplifiers that are being run in saturation for efficiency. Thus, different techniques are often needed for transmit beamforming.

We consider below design approaches for three types of transmit array pattern: directional (high gain), omnidirectional, and sector beams. We will see that the circular geometry poses both some challenges as well as some unique capabilities.

1) Directional Patterns: On transmit, the far-field effective radiated power (ERP) in the polarization direction $\hat{c}$ is proportional to the array pattern magnitude squared: $\mid \vec{F}\left(\mathbf{k}_{\mathrm{mb}}\right)$. $\left.\hat{c}\right|^{2}=\left|\mathbf{w}^{T} \mathbf{f}_{\mathrm{mb}}\right|^{2}$. If we assume well-matched transmitters and elements in the array, then the power fed to each element is proportional to the corresponding weight squared, and thus total input power is proportional to $\|\mathbf{w}\|^{2}$. The ratio of ERP to input power,

$$
\frac{\left|\mathbf{w}^{T} \mathbf{f}_{\mathrm{mb}}\right|^{2}}{\|\mathbf{w}\|^{2}}
$$

represents the gain of the array with respect to an isotropic radiator. Comparing to the last section, we see that this is exactly the expression (2) for SNR on receive; thus absent other constraints the set of weights $\mathbf{w}=\alpha \mathbf{f}_{\mathrm{mb}}^{*}$ will optimize transmit gain, with $\alpha$ a nonzero complex scalar whose magnitude determines the ERP.

In practice, radar transmitters are power-limited devices; this places constraints on the range of acceptable weights. This might take the form of a limit on total power, such that $\|\mathbf{w}\|^{2} \leq N \beta^{2}$ for some $\beta$. More commonly each element in an array is individually power limited, resulting in the auxilliary constraints $\left\{\left|w_{n}\right| \leq \beta\right\}$ for $n=1 \ldots N$. Often the transmitters run in saturation for efficiency and no amplitude control is available at all; we then say that the weights are phase only, and enforce the constraints $\left\{\left|w_{n}\right|=\beta\right\}$. Under these constraints, the maximum gain and maximum ERP solutions can differ. Consider the following set of optimization 
problems:

$$
\begin{aligned}
& \max _{\mathbf{w}} \frac{\left|\mathbf{w}^{T} \mathbf{f}_{\mathrm{mb}}\right|^{2}}{\|\mathbf{w}\|^{2}}, \text { s.t. : }\|\mathbf{w}\|^{2} \leq N \beta^{2} \\
& \max _{\mathbf{w}}\left|\mathbf{w}^{T} \mathbf{f}_{\mathrm{mb}}\right|^{2}, \text { s.t. : }\|\mathbf{w}\|^{2} \leq N \beta^{2} \\
& \max _{\mathbf{w}} \frac{\left|\mathbf{w}^{T} \mathbf{f}_{\mathrm{mb}}\right|^{2}}{\|\mathbf{w}\|^{2}} \text {, s.t. : }\left|w_{n}\right| \leq \beta, n=1 \ldots N \\
& \max _{\mathbf{w}}\left|\mathbf{w}^{T} \mathbf{f}_{\mathrm{mb}}\right|^{2} \text {, s.t. : }\left|w_{n}\right| \leq \beta, n=1 \ldots N \\
& \max _{\mathbf{w}} \frac{\left|\mathbf{w}^{T} \mathbf{f}_{\mathrm{mb}}\right|^{2}}{\|\mathbf{w}\|^{2}}, \text { s.t. : }\left|w_{n}\right|=\beta, n=1 \ldots N \\
& \max _{\mathbf{w}}\left|\mathbf{w}^{T} \mathbf{f}_{\mathrm{mb}}\right|^{2}, \text { s.t. : }\left|w_{n}\right|=\beta, n=1 \ldots N
\end{aligned}
$$

Here (6) and (7) optimize transmit gain and ERP, respectively, for total-power limited transmitters, while (8) and (9) optimize gain and ERP for peak-limited transmitters and (10) and (11) optimize gain and ERP for phase-only transmitters. The solutions to (6) and (7) are identical and have the form of the unconstrained solution with a particular value of $\alpha$ :

$$
\mathbf{w}=\frac{\sqrt{N} \beta}{\left\|\mathbf{f}_{\mathrm{mb}}\right\|} \mathbf{f}_{\mathrm{mb}}^{*} .
$$

The solution to (8) also has the same form as the unconstrained solution, but with a different scaling:

$$
\mathbf{w}=\frac{\beta}{\left\|\mathbf{f}_{\mathrm{mb}}\right\|_{\infty}} \mathbf{f}_{\mathrm{mb}}^{*}
$$

where $\left\|\mathbf{f}_{\mathrm{mb}}\right\|_{\infty}$ is the $l_{\infty}$ norm of $\mathbf{f}_{\mathrm{mb}}$. Hölder's inequality can be used to show that

$$
\sqrt{N}\left\|\mathbf{f}_{\mathrm{mb}}\right\|_{\infty} \geq\left\|\mathbf{f}_{\mathrm{mb}}\right\|,
$$

thus the scale factor in (12) is always greater than or equal to that in (13) and the peak-power constraints reduce ERP. The maximum ERP problem of (9) has solutions of the form

$$
w_{n}=\frac{\beta}{\left|\left[\mathbf{f}_{\mathrm{mb}}\right]_{n}\right|}\left[\mathbf{f}_{\mathrm{mb}}^{*}\right]_{n}, n=1 \ldots N
$$

in which each weight is driven to its magnitude limit. Since this solution meets the phase-only constraints $\left\{\left|w_{n}\right|=\beta\right\}$ as well, and since the constraint of (10) implies $\|\mathbf{w}\|^{2}=N \beta^{2}$, we see that (14) is the common solution to (9)-(11). These various solutions will be compared in the next section.

A drawback of using a UCA for directional beams is that for any given direction the majority of the elements are pointed elsewhere, and if driven may only provide marginal contribution to the mainbeam while elevating the sidelobes. There are are at least a couple of potential approaches to mitigate this, including driving only a subset of elements and forming more than one directional beam at a time. The former will be explored further in the examples.

2) Omnidirectional Patterns: A unique capability of a UCA is the ability to synthesize patterns that are omnidirectional in azimuth using the full power of each element. Such patterns can be used for so-called ubiquitous radar [8] which pairs omni transmit beams with multiple simultaneous directional receive beams to search the whole sky continuously instead of sequentially. Such patterns can be designed heuristically, or numerical optimization can be used in order to impose additional constraints. Examples of such constraints include imposing narrow notches to avoid interference with another platform, and to shape the elevation profile of the beam.

One approach to synthesizing phase-only omnidirectional patterns with multiple nulls while enforcing a phase-only constraint is outlined in [16], which solves the optimization

$$
\begin{aligned}
\min . & \left(\left.\int W^{4}(\mathbf{k})|| \vec{F}\left(\mathbf{k}_{\mathrm{mb}}\right) \cdot \hat{c}\right|^{2}-\left.D^{2}(\mathbf{k})\right|^{2} d \mathbf{k}\right)^{\frac{1}{2}} \\
\text { s.t. } & \left|w_{n}\right|=\beta, n=1 \ldots N .
\end{aligned}
$$

The objective is a weighted pattern error, where $D$ is the desired pattern magnitude and $W$ is a nonnegative weighting function that allows selective emphasis on different regions of the pattern. The desired magnitude pattern value is set to the desired gain value over the desired omnidirectional region, while $D$ is set to zero in desired null regions and for high elevation angles to reduce unwanted radiation. The values of $W$ for these regions are then set to achieve the desired pattern effects.

3) Sector Patterns: Directional and omnidirectional patterns represent the extremes in transmit beamwidth; in between lie sector beams covering an intermediate range of azimuth angles. Such beams will have higher gain than an omnidirectional beam and lower than a directional beam, and thus offer a continuum of options to trade off coverage and sensitivity. Sector beams from a UCA can be optimized via the same approach used in the previous section for omnidirectional beams, with suitable modifications to $D$ and $W$.

As is the case for directional beams, exciting directional elements that point far away from the desired sector can degrade the achievable SLL without significantly improving the sector gain. The same options are available: excite only a subset of the elements, or synthesize multiple sectors at once in order to efficiently use all the elements.

\section{EXAMPLE}

As an example, we synthesize patterns for the $N=31$ element circular array of circular patch elements shown in Fig. 1. The array has a 3 inch radius and operates at $10.0 \mathrm{GHz}$, which yields half-wavelength linear spacing between elements. The $\hat{z}$-axis is the axis of the array. The polar angle $\phi$ is measured from the $\hat{x}$-axis, and $\theta$ is measured down from the $\hat{z}$ axis. The prototype embedded element pattern is obtained by exciting a single element of the full array in CST Microwave Studio, and the remaining embedded element patterns are determined using (1).

We first design an omnidirectional pattern with 2 nulls at $\pm 45^{\circ}$. The transmit pattern shown in Fig. 2 is synthesized using the technique from Sec. II-B2. Here, the optimized pattern is shown in blue and the desired pattern magnitude $D$ is shown in red. The omnidirectional regions of this pattern have more than desired ripple which is attributed to the degrees of freedom taken up by the nulls. Adding extra elements (while maintaining the same radius) would help mitigate this effect. This pattern shape would be convenient for a radar system tasked with illuminating the entire azimuth plane with the 


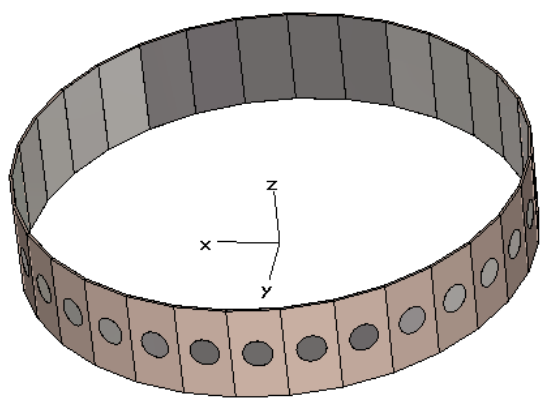

Fig. 1. 31-element circular array used in example calculation.

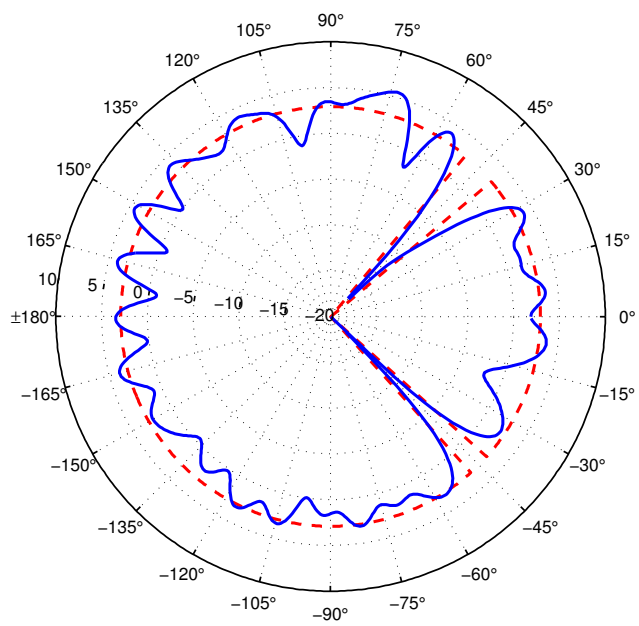

Fig. 2. Directivity ( $\mathrm{dBi}$ ) of the synthesized omnidirectional pattern with 2 nulls regions covering $43^{\circ} \leq \phi \leq 47^{\circ}$ and $314^{\circ} \leq \phi \leq 316^{\circ}$. Each region was bounded by $80^{\circ} \leq \theta \leq 100^{\circ}$. The synthesized pattern and desired pattern are shown.

exception of areas where it must reduce interference. If the interference must be reduced over a wider angular region, the radar system could use the formulation from Sec. II-B3 to form a sector pattern as shown in Fig. 3. This example sector pattern is centered at $\phi=180^{\circ}$ with an angular width of $120^{\circ}$. The desired magnitude pattern - overlaid in red has a magnitude of $4.0 \mathrm{~dB}$ within the sector and 0.0 outside of the sector. This sector pattern was formed using only 11 elements, allowing the remaining elements to be used for other transmit functions. For example, one could create three such $120^{\circ}$ beams to illuminate each sector independently.

If the radar system has to maximize its power on target, it is desirable to transmit directional patterns from the circular array. Here, we consider four cases: the matched weights solution of (12), the peak-limited weights solution of (13), the phase-only weights solution of (14), and the phase-only weights with only an 11-element subset excited. We assume a normalized total power limit of $\|\mathbf{w}\|^{2} \leq 1$, with individual element weights limited to $\left|w_{n}\right| \leq 1 / \sqrt{N}$ for the peakconstrained case and $\left|w_{n}\right|=1 / \sqrt{N}$ for the phase-only cases.

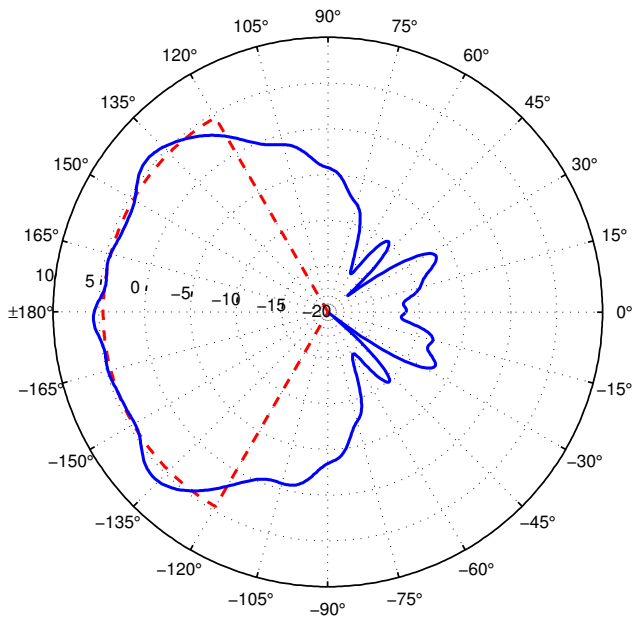

Fig. 3. Directivity (dBi) of synthesized $120^{\circ}$ sector pattern centered at $\phi=$ $180^{\circ}$. The synthesized pattern and desired pattern are shown.

The resulting gain, input power, and ERP for these four cases are compared in Table I, with the array patterns shown in Fig. 4 and the element excitation magnitudes shown in Fig. 5.

Of these four designs, the matched weights case has the maximum gain, input power, and ERP; it represents the most efficient distribution of power across the array. Limiting the peak excitation magnitude to $1 / \sqrt{N}$ has no effect on the gain but reduces total input power (and thus ERP) by over $5 \mathrm{~dB}$. The phase-only weights have the same input power as the matched weights excitation, but distributed evenly across the array. This excitation, while having a lower ERP and gain than the matched weights excitation, addresses practical limitations for a radar transmitter where it becomes desirable to run all power amplifiers in saturation. A drawback of exciting all elements in a circular geometry to produce a single directional beam is that the elements with pointing angles far from the desired boresite direction contribute little to the ERP while raising the sidelobes and the back lobe of the array pattern. To mitigate this, we can drive only a subset of the array elements as shown in the case where only 11 of the 31 elements are excited. In doing so, we have reduced the input power by $4.5 \mathrm{~dB}$, but the gain of the directional pattern has increased by $0.9 \mathrm{~dB}$. This excitation configuration is also attractive because it allows the radar to use the remaining elements to form additional simultaneous beams.

Regardless of the transmit beam shape, it's advantageous for the radar system to be able to form multiple closelyspaced beams on receive. If the array has element-level digital receivers, the SOCP-generated weights from Sec. II-A can be applied to optimize weights for arbitrarily-pointed receive beams. This is illustrated in Fig. 6, where we show 36 receive beams that cover the entire azimuth plane and overlap at their $3 \mathrm{~dB}$ points. These receive patterns were designed to minimize taper loss without any additional constraints to SLL. These beams can be generated at arbitrary locations in azimuth spaced as closely together as desired - with only minimal 


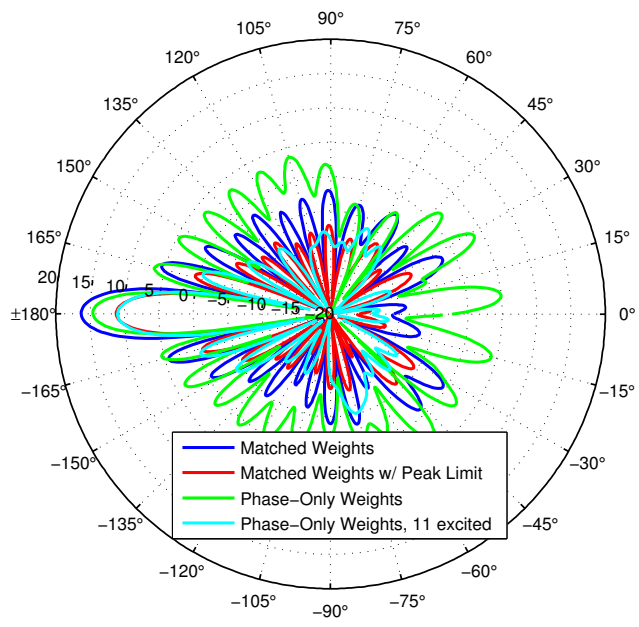

Fig. 4. Effective radiated power $(\mathrm{dB})$ of synthesized directional patterns with beam peak at $\phi=180^{\circ}$ using the multiple techniques outlined in Sec. II-B1.

TABLE I

GAIN, INPUT POWER, AND EFFECTIVE RADIATED POWER (ERP) FOR THE PATTERNS SHOWN IN FIG. 4

\begin{tabular}{|c|c|r|c|}
\hline Excitation Type & Gain & Input Power & ERP \\
\hline \hline Matched Weights & $16.3 \mathrm{dBi}$ & $0.0 \mathrm{~dB}$ & $16.3 \mathrm{~dB}$ \\
\hline Peak-Limited Weights & $16.3 \mathrm{dBi}$ & $-5.1 \mathrm{~dB}$ & $11.2 \mathrm{~dB}$ \\
\hline Phase-Only Weights & $14.6 \mathrm{dBi}$ & $0.0 \mathrm{~dB}$ & $14.6 \mathrm{~dB}$ \\
\hline Phase-Only Weights (11 excited) & $15.5 \mathrm{dBi}$ & $-4.5 \mathrm{~dB}$ & $11.0 \mathrm{~dB}$ \\
\hline
\end{tabular}

changes seen to beam width and SLL.

\section{CONCLUSION}

This paper outlines pattern synthesis techniques that facilitate the integration of a uniform circular array into a radar system. On transmit, we apply a phase-only pattern synthesis approach that uses an efficient unconstrained formulation. We have demonstrated the ability to efficiently form omnidirectional, sector, and directional beams. On receive, we have formulated the problem as a second-order cone program to form directional beams with appropriate constraints on taper loss and sidelobe level. These pattern synthesis techniques allow a radar system to take full advantage of the pattern flexibility offered by a uniform circular array.

\section{REFERENCES}

[1] J.-A. Tsai and B. Woerner, "Performance of diversity combining for uniform circular arrays," in Vehicular Technology Conference, 2001. VTC 2001 Fall. IEEE VTS 54th, vol. 3, 2001, pp. 1630-1634 vol.3.

[2] Q. Shen, E. ke Mao, and W. Si-liang, "The performance analysis of circular array antennas in vhf/uhf band," in International Conf. on Radar, 2006, pp. 1-4.

[3] E. Yildirim and E. Ercil, "Development of an 1 band cylindrical phased array," in IEEE Symp. on Phased Array Systems Technology, Oct 2013, pp. $746-751$.

[4] M. Malanowski and K. Kulpa, "Digital beamforming for passive coherent location radar," in IEEE Radar Conference, May 2008, pp. 1-6.

[5] B. Jackson, S. Rajan, B. Liao, and S. Wang, "Direction of arrival estimation using directive antennas in uniform circular arrays," IEEE Trans. Antennas Propag., vol. 63, no. 2, pp. 736-747, Feb 2015.

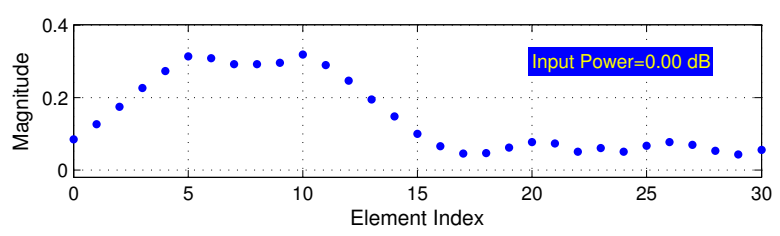

(a) Matched Weights

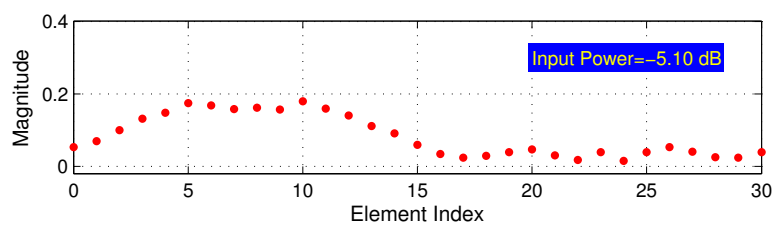

(b) Peak-Limited Weights

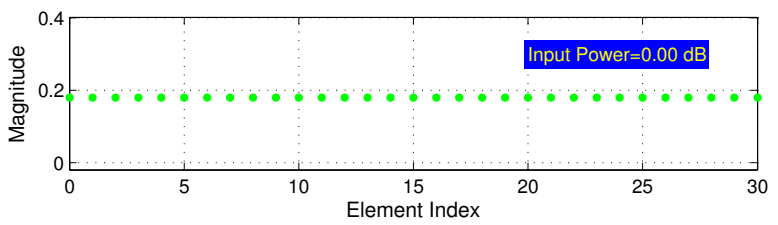

(c) Phase-Only

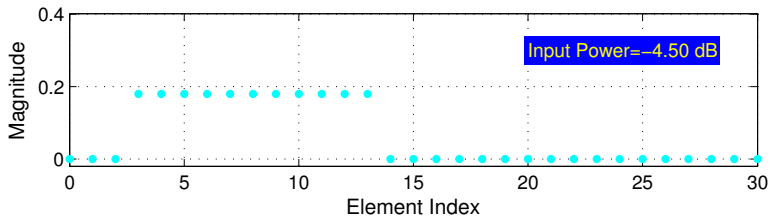

(d) Phase-Only (11 excited)

Fig. 5. Amplitudes of the element excitations used to synthesize the four patterns shown in Fig. 4.

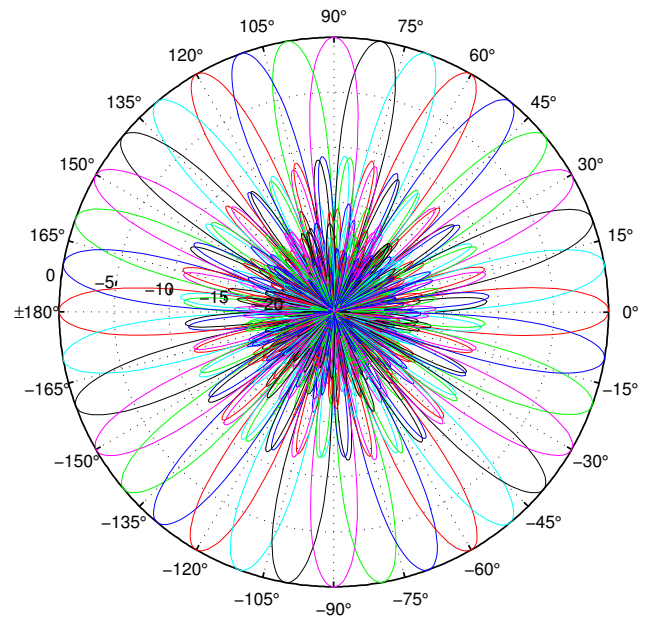

Fig. 6. Synthesized receive beams formed using the SOCP technique of Sec. II-A. 36 beams - each having a $10.0^{\circ}$ beamwidth - overlap at their $3.0 \mathrm{~dB}$ points to cover the entire azimuth plane. 
[6] W. Dorsey, J. Coleman, and W. Pickles, "Uniform circular array pattern synthesis using second-order cone programming," IET Trans. on Microwaves, Antennas Propagation, vol. 9, no. 8, pp. 723-727, 2015.

[7] G. Brown, J. Clayton Kerce, and M. Mitchell, "Extreme beam broadening using phase only pattern synthesis," in IEEE Workshop on Sensor Array and Multichannel Processing, July 2006, pp. 36-39.

[8] M. Skolnik, "Attributes of the ubiquitous phased array radar," in IEEE Int'l Symp. Phased Array Systems and Technology, Oct 2003, pp. 101106.

[9] W. Dorsey and D. Scholnik, "A hybrid global-local optimization approach to phase-only array-pattern synthesis," in IEEE Radar Conference (RadarCon), May 2015, pp. 1417-1422.

[10] D. Kelley and W. Stutzman, "Array antenna pattern modeling methods that include mutual coupling effects," IEEE Trans. Antennas Propag., vol. 41, no. 12, pp. 1625-1632, 1993.

[11] T. Rahim and D. Davies, "Effect of directional elements on the directional response of circular antenna arrays," IEE Proc. on Microwaves, Optics and Antennas, vol. 129, no. 1, pp. 18-22, February 1982.

[12] J. F. Sturm, "Using SeDuMi 1.02, a MATLAB toolbox for optimization over symmetric cones," in Optimization Methods and Software, 1999. vol. 11-12, pp. 625-653, special issue on Interior Point Methods.

[13] K. Toh, M. Todd, and R. Tutuncu, "SDPT3-a matlab software package for semidefinite programming," Optimization Methods and Software, vol. 11 , pp. 545-581, 1999.

[14] A. Domahidi, E. Chu, and S. Boyd, "ECOS: an SOCP solver for embedded systems," European Control Converence, 2013. [Online]. Available: http://www.stanford.edu/ boyd/papers/pdf/ecos_ecc.pdf

[15] D. P. Scholnik, "A parameterized pattern-error objective for large-scale phase-only array pattern design," IEEE Trans. Antennas Propag., 2015, to be published.

[16] W. Dorsey, R. Mital, and D. Scholnik, "Phase-only synthesis of omnidirectional patterns with multiple nulls from a uniform circular array," in IEEE Ant. and Prop. International Symp., vol. Submitted, 2016. 